Sharif University of Technology
Scientia Iranica
Transactions A: Civil Engineering
SCIENTIA
IRAN ICA

\title{
Modal data-based approach to structural damage identification by means of imperialist competitive optimization algorithm
}

\author{
H. Dabbagh ${ }^{a, *}$, G. Ghodrati Amiri ${ }^{b}$, and Sh. Shaabani ${ }^{a}$ \\ a. Department of Civil Engineering, University of Kurdistan, Sanandaj, Iran. \\ b. Center of Excellence for Fundamental Studies in Structural Engineering, School of Civil Engineering, Iran University of Science \\ \& Technology, Tehran, Iran.
}

Received 6 March 2016; received in revised form 22 October 2016; accepted 11 November 2017

\section{KEYWORDS}

Structural damage

detection;

Model updating;

ICA;

Modal assurance

criterion.

\begin{abstract}
In this paper, an effective method for structural damage detection is put forth in which an objective function based on the natural frequencies and modal shapes of the structure is established to identify and detect structural damage. The problem is defined and solved as an optimization problem employing Imperialist Competition Algorithm (ICA). Moreover, four numerical examples are examined each of which has different damage scenarios in order that the applicability of the method would be clearly proved. The results show the efficiency of the method in detecting single and multiple damages to different structures. Afterwards, the effects of measurement noises are included in some of the examples so that the method can be more consistent with real situations. Besides, a comparison among several evolutionary optimization algorithms in the research is made to enlighten the accuracy, robustness and reliability of the method. All of the results lead to the conclusion that the suggested method of the paper is of good accuracy and, therefore, can be both used and trusted in solving damage detection problems, even in the case of measurement noises.
\end{abstract}

(C) 2018 Sharif University of Technology. All rights reserved.

\section{Introduction}

Structural damages normally occur due to different loading and environmental conditions which affect the design's service life and desired performance of the structures. In order to identify such defects and prevent their progress, structural damage detection methods have been developed. More importantly, detecting structural damages in the earliest stage is the most important aspect of this branch of structural health monitoring to which a large amount of literature

*. Corresponding author.

E-mail address: h.dabbagh@uok.ac.ir (H. Dabbagh). is devoted. Amongst different methods offered so far, vibration-based structural damage detection is of greatest importance due to its simple implementation as well as convenient measurement feasibility. A comprehensive review of damage detection via vibration methods is given in $[1,2]$. Generally, structural damages alter the modal properties of the structure which can be conducive to finding such defects [38]. Modal data-based methods of detecting damage have been developed by different researchers [9-13]. The most common modal data applied are natural frequencies and modal shapes [14-24].

Natural frequency has the advantage of being easily measured for structures, and it is not dependent on the position of the measurement instruments. Moreover, measurements of natural frequencies are 
of higher accuracy than those of modal shapes are. A structural damage detection scheme is established using the natural frequencies of the structures by Kaveh and Zolghadr [25], where the authors solve the inverse problem of damage detection using changes of natural frequencies. They also use the Charged System Search (CSS) algorithm to solve the optimization problem of damage detection and exemplify their method for a two-span beam, a two-span three-story frame and a four-span five-story frame. In [26], a vibrationbased damage detection scheme employing the natural frequencies of the structure was proposed and great efforts were made so as to overcome the obstacles faced while utilizing the natural frequencies, where a Particle Swarm Optimization (PSO) algorithm was applied to optimize the inverse problem of damage identification. However, this parameter does not provide enough information to completely identify and detect any damage to the structure. Furthermore, we need to detect both the severity and the location of damage, and this cannot be accomplished by using natural frequencies only, since relatively high damage severities cause only small changes in natural frequencies; thus, small damages which possess a greater degree of importance in global damage detection problems cannot be detected by examining the natural frequencies alone.

Apart from natural frequencies, measuring vibration mode shapes of the structures can be helpful for detecting structural damage since mode shapes contain a great amount of information about the damages to the structure. In this context, a damage detection approach using the modal shapes was elevated by Kaveh et al. [27], where an objective function based on mode shapes was defined, and the problem was optimized using a mixed PSO, ray optimizer, and harmony search. Furthermore, a two-stage method for detecting damage in high-rise shear frames was proposed based on the first mode shape slope in [28] where the optimization problem was solved via ICA. Other scholars, on the other hand, are available which suggest using modal shape curvatures so as to identify structural damage. Pandey et al. [29] investigated the existence as well as the location and severity of structural damage in two beams by comparing curvature mode shapes of the elements; their research was shown to be successful in this respect. There are also a wide variety of literature investigating structural damage using different modal parameters of the structure. Moreno-Garcia et al. [30], however, adopted a new technique based on Ritz method so as to estimate the computation error of mode shape derivatives of structures, by which the optimal spatial sampling can be defined to minimize the influence of the error. They compute first-, second-, third-, and fourth-order derivatives using finite differences with a quadratic convergence of the spatial sampling in order to localize damage in a laminated composite plate.

In [31], a damage detection scheme was provided aiming to determine structural damage by investigating the changes in the flexibility matrix of the structure. Balsamo et al. [32], on the other hand, defined a modal parameter-based damage sensitive feature which imitates the relative changes in diagonal elements of the stiffness matrix of a structure. The damage assessment in their research was carried out on a statistical framework using empirical complementary cumulative distribution functions of the damage sensitive features which, themselves, are derived from measured operational vibration response data. In [33], a numerical model was proposed for damage identification of composite structures modeled based on orthogonality condition sensitivities of the structures' mode shapes in both damaged and undamaged states.

To derive benefits from both natural frequencies and mode shapes simultaneously, Perera et al. [34] employed a combination of natural frequencies and mode shapes of the structure in a multi-objective framework to construct an objective function for damage detection. Instead of directly using these parameters, they make use of their differences in the objective function. They proved in the paper that the differences of modal shapes and natural frequencies are not sensitive to modeling errors and are damage-sensitive parameters. Although their method seems effective, there are discrepancies in the approximations made for the damaged elements; more precisely, damage is not exactly determined. Later on, utilizing the first few natural frequencies as the main parameters of the objective function and benefiting from multistage PSO as the optimizer to solve the inverse problem, a new damage detection method was proposed in [35]. In [36], an objective function based on natural frequencies and modal shapes of the structure was defined via the Modal Assurance Criterion where the inverse optimization problem is solved via employing a fusion of the GA and PSO. The performance of the paper is then assessed by the authors using a number of numerical studies demonstrating the performance of their suggested method. A Magnetic Charged System Search (MCSS) and a PSO are applied to the problem of damage detection using frequencies and mode shapes of the structures through which the performance of the process is validated using a variety of numerical examples in [37]. Seyedpoor and Yazdanpanah [38] solved the problem of damage detection by applying the Differential Evolution (DE) as a global optimizer with an objective function defined based on the changes in frequency vectors. More recently, a structural damage detection scheme has been proposed in [39] where frequency residual error and the modal assurance criterion were used as damage 
indices and the cuckoo search was utilized as the optimizer.

According to what is stated above, a new objective function is proposed in this paper which consists of both natural frequencies and mode shapes in order to enhance the accuracy and efficiency of the damage detection algorithm. Therefore, severity and location of structural damage can be detected with great accuracy using the method proposed; further, damage detection problem is defined as an optimization problem with an objective function assigned using the Modal Assurance Criterion (MAC), to be minimized. The parameters used in MAC are natural frequencies and modal shapes of the structure. The Imperialist Competition Algorithm (ICA) is then used to optimize the objective function; in this way, the process of detecting damage is terminated. The method has been validated using four numerical examples including two beams: a shear frame and a plane truss. The results emphasize good and viable applicability of the presented method in damage identification.

\section{Material and methods}

\subsection{Objective function}

To achieve the proposed purpose, an appropriate objective function has to be defined that consists of a number of parameters which are sensitive enough to detect all of the probable damages in the structure. The start point is the free vibration equation of the structures:

$$
\left(\left[\mathbf{K}_{d}\right]-\left(\lambda_{j}^{d}\right)[\mathbf{M}]\right)\left\{\boldsymbol{\Phi}_{j}^{d}\right\}=0, \quad j=1, \ldots, N_{m},
$$

where $\left[\mathbf{K}_{d}\right]$ is the global stiffness matrix of the damaged structure, $[\mathbf{M}]$ is the global mass matrix of the structure, $\lambda_{j}^{d}$ represents the squared natural frequencies of the damaged structure corresponding to the $j$ th vibration mode, $\boldsymbol{\Phi}_{j}^{d}$ represents the mode shapes of the damaged structure, and $N_{m}$ is the total number of modes considered. $[\mathbf{M}]$ is assumed to be unchangeable during the process of damage, that is:

$$
[\mathbf{M}] \approx\left[\mathbf{M}_{d}\right] .
$$

Damage is modeled in the form of reduction in the stiffness of the structure and is mathematically shown in Eq. (3) where scalar variable, $d$, the values of which are bounded between 0 and 1, quantifies damage:

$$
\left[\mathbf{k}_{d}^{e}\right]=\left(1-d_{e}\right)\left[\mathbf{k}^{e}\right],
$$

where $\left[\mathbf{k}^{e}\right]$ and $\left[\mathbf{k}_{d}^{e}\right]$ are the eth element's stiffness matrices of the intact and damaged structures, respectively. $\left[\mathbf{k}_{d}\right]$ is obtained through the assemblage of $\left[\mathbf{k}_{d}^{e}\right]$. Scalar $d$ takes a zero value when there is no damage, and the values near one show complete rupture.

As mentioned before, this paper is aimed at presenting a novel objective function with high level of sensitivity to structural damages. This function is defined as follows:

$$
\begin{aligned}
& F=F_{1} \times \prod_{j=1}^{N} F_{2_{j}}, \\
& F_{1}=\frac{1-\mathrm{MAC}_{\omega}}{1+\mathrm{MAC}_{\omega}} \\
& F_{2_{j}}=\frac{1-\mathrm{MAC}_{\phi_{j}}}{1+\mathrm{MAC}_{\phi_{j}}},
\end{aligned}
$$

where $\mathrm{MAC}_{w}$ is the MAC value of the natural frequencies, and $\mathrm{MAC}_{\phi_{j}}$ is that of the $j$ th mode shape. Therefore, the introduced objective function measures the correlation between the numerical natural frequencies generated by the model and those of the experimental data. The same is done for the mode shapes of the structure. In other words, the MAC value is calculated for the numerical quantities of natural frequencies as well as mode shapes of the structure and those of the experimental data to see the degree of correlation between these two data-sets. This will result in finding the best predictions of the damage locations and severities. The basic concepts of MAC are included as follows:

\subsubsection{Modal assurance criterion}

The Modal Assurance Criterion (MAC) is an essential tool in modal analysis. Because of its success in measuring the correlation between two vectors, this criterion has become a method of great importance. Conceptually, the Modal Assurance Criterion (MAC) is a measure of the degree of linearity between two vectors [40]. The modal assurance criterion is defined as follows:

$$
\operatorname{MAC}_{j}=\frac{\left|\left\{x_{j}^{d}\right\}^{T}\left\{x_{j}^{*}\right\}\right|^{2}}{\left(\left\{x_{j}^{d}\right\}^{T}\left\{x_{j}^{d}\right\}\right)\left(\left\{x_{j}^{*}\right\}^{T}\left\{x_{j}^{*}\right\}\right)}
$$

where $\left\{x_{j}^{d}\right\}$ represents the numerical vectors generated during the modal analysis and optimization process and $\left\{x_{j}^{*}\right\}$ refers to experimental vectors.

Vectors used here to assess MAC values are the natural frequencies and mode shapes of the structure. Hence, when the first ' $m$ ' modes are used for solving the presented problem, the natural frequency-based term in Eq. (4) consists of the first ' $m$ ' modes' frequencies, and the mode shape-based term can be formed by employing the first ' $m$ ' mode shape vectors.

\subsection{Optimization techniques}

2.2.1. Imperialist competition algorithm

Imperial is the word for a government whose power is extended beyond its own physical boundaries. Such 
countries always try to increase the number of their colonies in order to extend their power all over the world. Therefore, there is an inherent competition among imperialist countries which enhances the power of the stronger empires and further weakens that of the relatively weaker ones. Inspiring these political rules, the ICA has been developed, which is an evolutionary optimization algorithm, to find an optimal solution, usually the minimum of argument $X$ of a certain function, $f(x)$. Similar to other evolutionary optimization algorithms, this algorithm starts with an initial population called country. The number of countries is equal to $N_{\text {country }}$, and they are divided into $N_{\text {imp }}$ imperialists and $N_{\text {col }}$ colonies. Every country is presented in a vector as follows [41]:

$$
\text { country }=\left[p_{1}, p_{2}, \ldots, p_{n}\right] .
$$

The value of the objective function obtained from a trial solution can be calculated by a function as follows:

$$
\operatorname{cost} t=f(\text { country })=f\left(p_{1}, p_{2}, \ldots, p_{n}\right) \text {. }
$$

The algorithm starts with an initial population of size $N_{\text {pop }}$. To divide the colonies among the empires with respect to their powers, the normalized cost of an empire is defined as follows:

$$
N C_{n}=c_{n}-\max _{i}\left\{c_{i}\right\},
$$

where $C_{n}$ is the cost of the $n$th empire. The colonies are assigned to empires based on their power, $P_{n}$ :

$$
P_{n}=\left|\frac{N C_{n}}{\sum N C_{i}}\right| \text {. }
$$

Then, the initial colonies are shared based on empires' normalized power as follows:

$$
\mathrm{NOC}_{n}=\operatorname{round}\left\{p_{n} \cdot\left(N_{\mathrm{col}}\right)\right\} \text {, }
$$

where $\mathrm{NOC}_{n}$ is the number of the colonies of the $n$th empire, and $N_{\text {col }}$ is the total number of colonies. After the initialization process, the imperialistic countries begin to improve their colonies and attempt to absorb new colonies. This is the assimilation process which is modeled by moving the colonies towards the imperialist along different optimization axes. A random path is induced by a random deviation added to the direction of the movement in order to better explore the search space.

The total power of an empire depends on both the power of the imperialist country and the power of its colonies. Therefore, the total cost of the $j$ th empire $T . C_{. j}$ is determined by:

$$
\begin{aligned}
T . C ._{j} & =f(\text { imperialist }) \\
+\xi & \frac{\sum_{i=1}^{N . C \cdot j} f(\text { the } i \text {-th colony of the } j \text {-th empire })}{N . C \cdot j},
\end{aligned}
$$

where $\xi$ is a coefficient between 0 and 1 . Normalized total cost N.T.C. of the $j$ th empire is obtained as follows:

$$
\text { N.T.C. }{ }_{j}=\text { T.C. } \cdot j-\max _{i}\left\{T . C \cdot{ }_{i}\right\} .
$$

Then, possession probability, $p_{p}$, of the $j$ th empire is given by:

$$
p_{p_{j}}=\mid \text { N.T.C.j } / \sum_{i=1}^{N_{\mathrm{imp}}} \text { N.T.C. } \cdot \mid .
$$

To share the colonies among the empires, vector $\mathbf{D}$ is defined as follows:

$$
\mathbf{D}=\mathbf{P}-\mathbf{R},
$$

where vector $\mathbf{D}$ is generated by random numbers uniformly distributed between 0 and 1 , and vector $P$ is defined as follows:

$$
\mathbf{P}=\left[p_{p_{1}}, p_{p_{2}}, \ldots, p_{p_{N_{i m p}}}\right] .
$$

A certain colony is given to an empire whose associated index in $D$ has the maximum value. The last step is the elimination of the weak and powerless empires. If only one empire is left, then the optimization algorithm is terminated; otherwise, the algorithm starts again from the assimilation step [36].

To summarize, the following steps ought to be executed in order that the ICA can be performed on any arbitrary optimization problem, a flowchart of which can be seen in Figure 1.

1. A number of points are selected randomly on the search space, thus initializing the empires;

2. The colonies are moved towards their associated imperialists (assimilation process);

3. If a colony associated with an empire possesses a lower cost than that of the imperialist, the positions of the colony and the imperialist ought to be switched;

4. The total cost of all of the empires is calculated;

5. The weakest colonies are taken from the weakest empires, and then are given to the most powerful empire;

6. The powerless empires are eliminated;

7. The algorithm is terminated if one single empire is left; otherwise, the algorithm is restarted from step 2.

In the context of damage detection, the cost function is the objective function defined by Eq. (4). In addition, a flowchart is presented in Figure 2 which illustrates different steps of the damage detection process in the framework for the optimization procedure. 


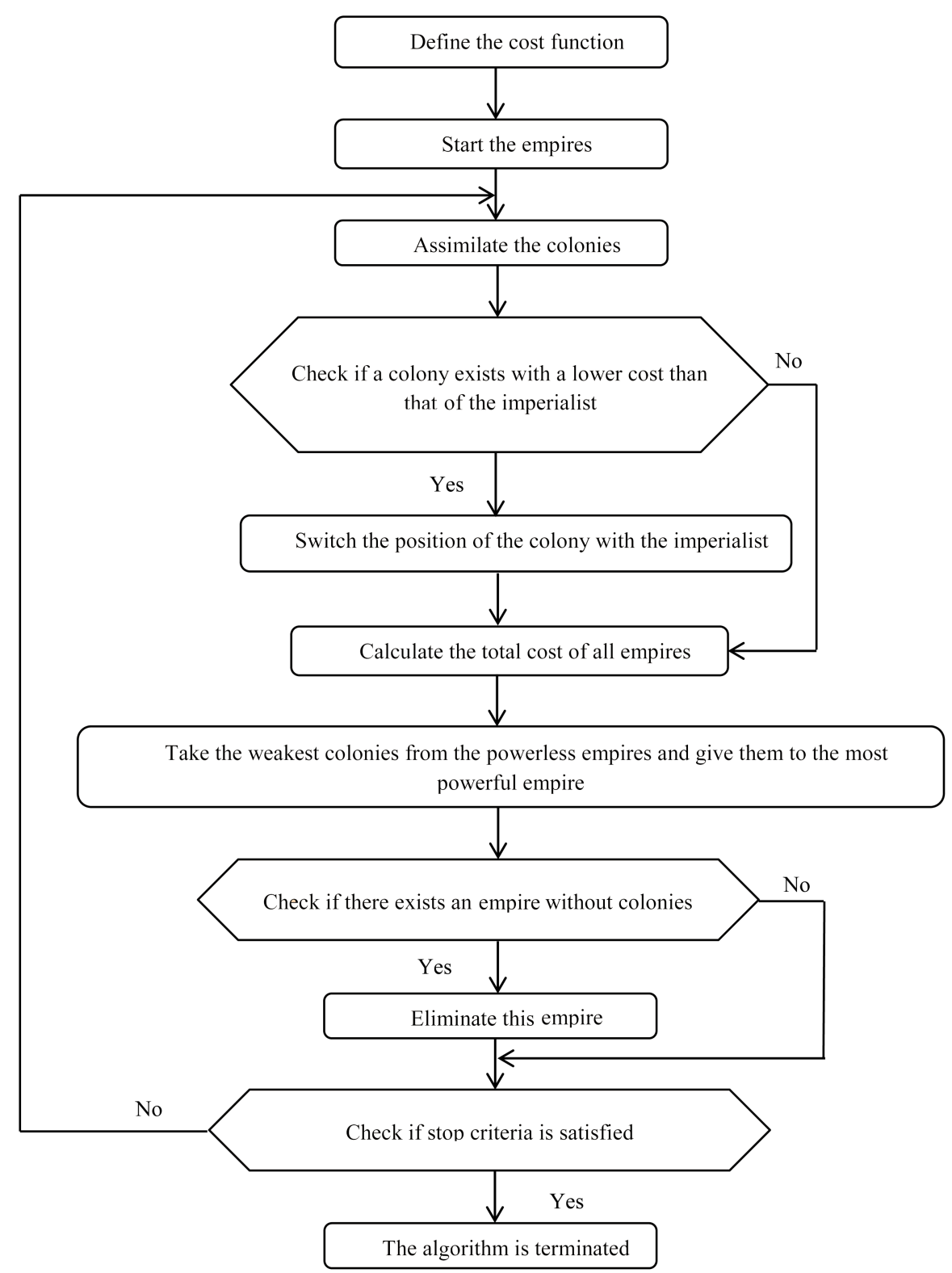

Figure 1. Flowchart of optimization using the imperialistic competition algorithm.

To be more precise, different steps and parameters of the ICA algorithm are associated with those of the damage detection problem so that the process can be more conveniently tracked in the optimization framework.

\section{Numerical study results and discussions}

This section is devoted to investigating the efficiency and applicability of the proposed approach to numerical examples. Thus, four numerical examples are examined in which simulations are made considering real-world obstacles such as measurement noise. In addition, the ICA solutions are compared to those of 3 other optimizers, and the results are included in order that the robustness of the method proposed by this paper can be clearly demonstrated. It is assumed in the paper that, notably, all active DOFs of the structures are measured via installed sensors. Accordingly, no incompleteness of data is observed during the damage detection process.

\subsection{Ten-story shear frame}

As a first example, a ten-story one-bay shear frame, as shown in Figure 3, has been studied. The physical properties of the frame are as follows: $K=2 \times$ $10^{6} \mathrm{~N} / \mathrm{m}, m=5 \times 10^{4} \mathrm{~kg}$. As listed in Table 1 , a damage scenario is considered for the frame. In order to make the situation simpler and more vivid for en gineering judgments, not only is the ideal case of noiseless state studied, but also the input measurement data are contaminated with noise and their effects are 


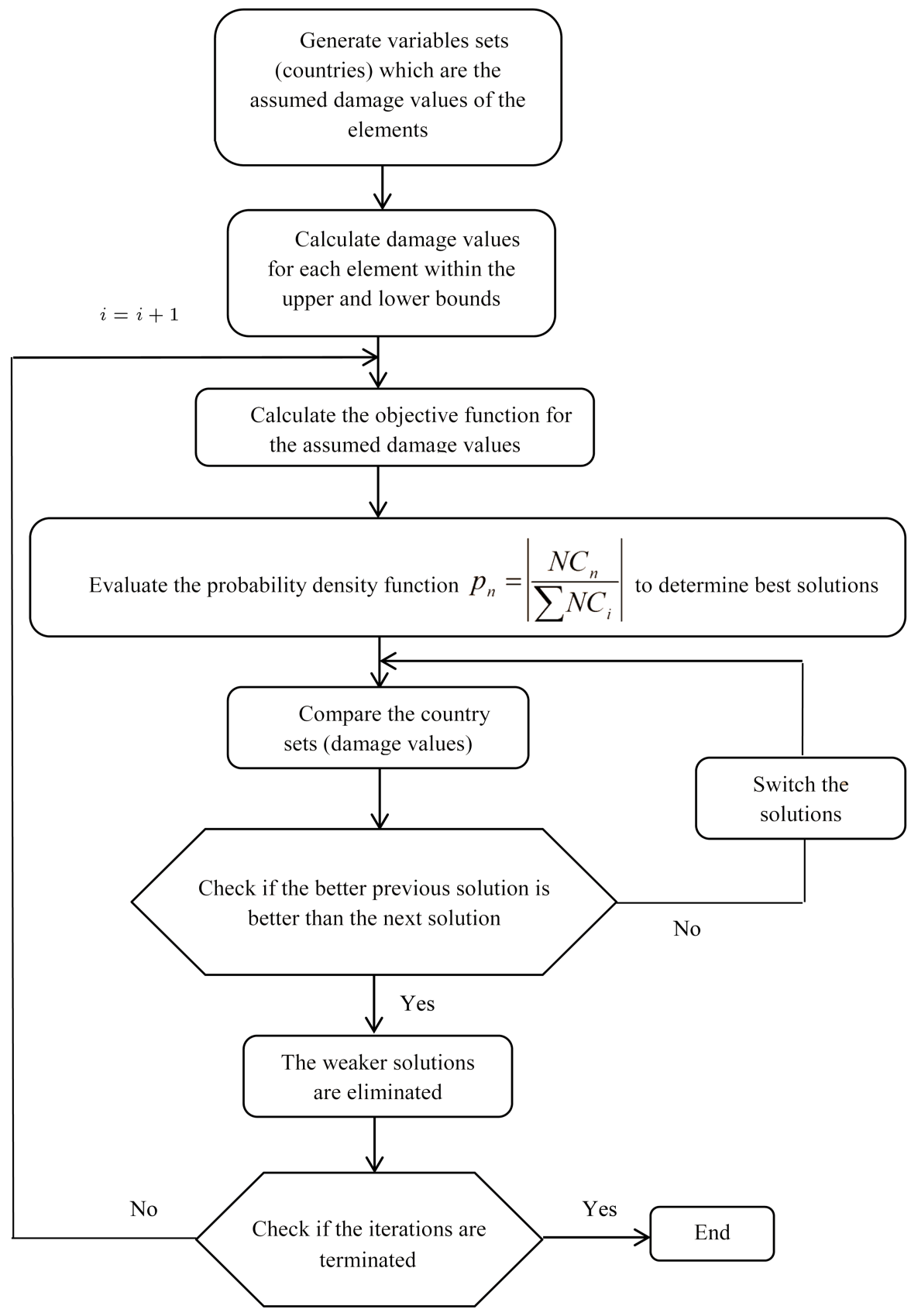

Figure 2. Flowchart of damage detection procedure via ICA.

Table 1. Damage scenarios for the 10-story shear frame.

\begin{tabular}{ccc}
\hline Scenario & $\begin{array}{c}\text { Element } \\
\text { number }\end{array}$ & $\begin{array}{c}\text { Damage } \\
\text { percent }\end{array}$ \\
\hline \multirow{2}{*}{1} & 3 & 5 \\
& 6 & 15 \\
\hline
\end{tabular}

taken into account in the computations. It is worth mentioning that the contaminating noise is added to the experimental data using the following equations as presented in [42]:

$$
\omega_{i}^{n}=\omega_{i}\left(1+\kappa \theta_{i}\right)
$$

$$
\phi_{i}^{n}=\phi_{i}\left(1+\varepsilon \eta_{i}\right)
$$

where $\omega_{i}^{n}$ and $\phi_{i}^{n}$ are the $i$ th natural frequency and mode shape vector; $\kappa$ and $\varepsilon$ are noise levels imposed on natural frequencies and mode shapes, respectively; $\theta_{i}$ and $\eta_{i}$ are a random value and a random vector generated between $[-1,1]$ via MATLAB, respectively.

As it can be observed from the table, element no. 3 is assumed to contain $5 \%$ damage, and element no. 6 contains $15 \%$ damage.

Another item the impacts of which are of interest is the number of measurement modes to be considered in the calculations. Thus, the problem is solved 


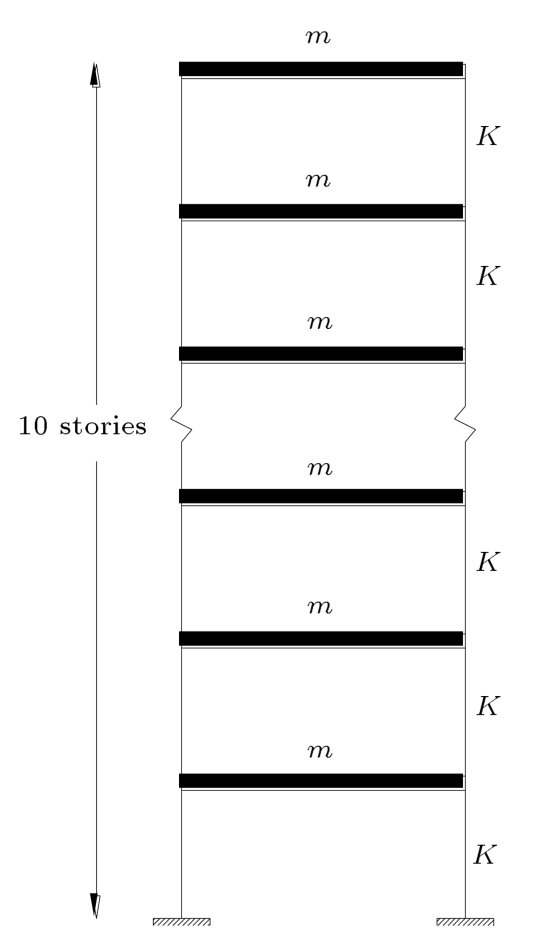

Figure 3. Ten-story shear frame.

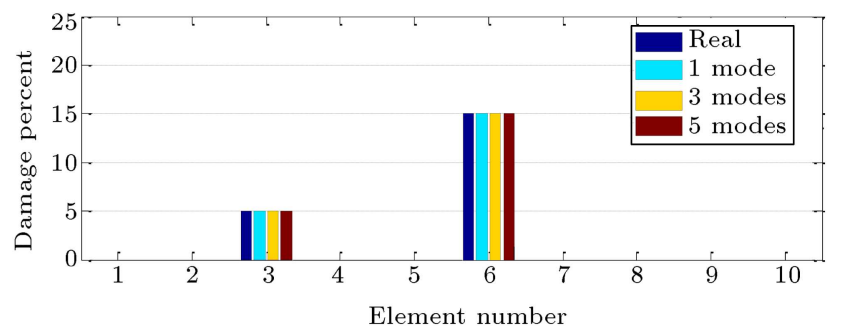

Figure 4. Damage detection results of the shear frame without noise.

considering 3 different cases in which the first 1,3 , and 5 vibration modes of the structure are considered. The problem is then optimized using the ICA, with selected parameters as follows: number of decades $=$ 500 , number of countries $=200$, and number of initial imperialists $=20$. It is worth noting that the associated parameters of the optimization algorithm are selected by trial and error since there is no overall rule dominating all optimization problems. The damage detection results of the noiseless state are shown in Figure 4.

It can be clearly observed from the figure that all results are in good agreement with the real damaged state; thus, it can be concluded that, regardless of the number of vibrational modes considered, structural damages are accurately detected with this method.

The above results were obtained for the noiseless case. To further see the efficiency of the method in real conditions, the results are obtained for the case in which frequency data are contaminated with $5 \%$ noise. Similar to the noiseless state, the results considering the first 1, 3, and 5 modes of the frame are acquired.

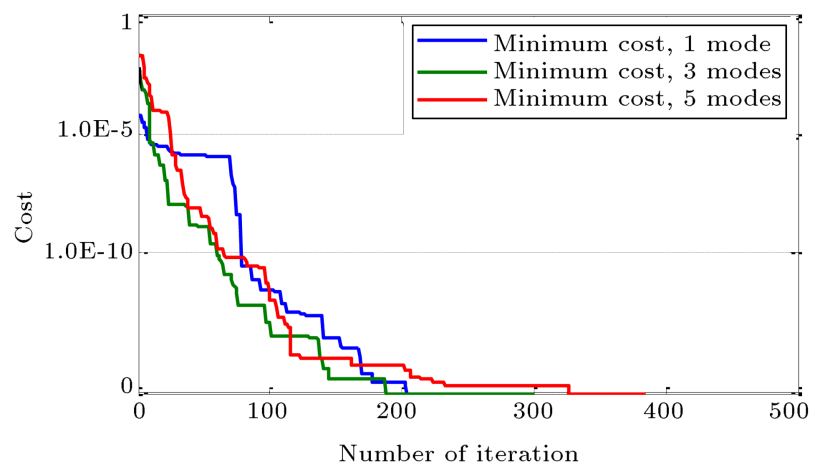

Figure 5. Cost function value versus number of iteration.

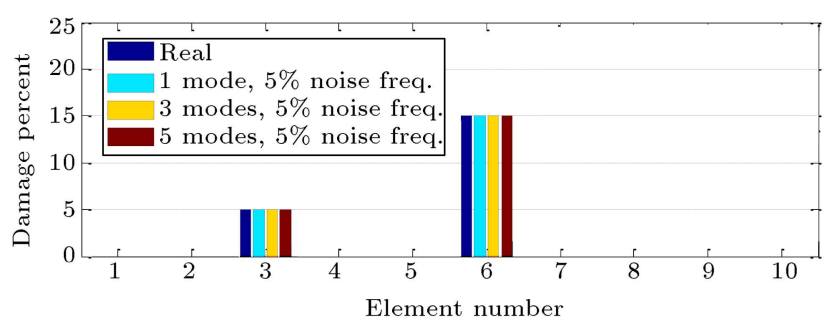

Figure 6. Damage detection results of the shear frame with $5 \%$ noise in $\omega$.

The problem is then optimized using the ICA, with selected parameters as follows: number of decades $=$ 500 , number of countries $=200$, and number of initial imperialists $=20$. The convergence curve for this case is shown in Figure 5, and damage detection results are shown in Figure 6.

As it can be seen from Figure 5, the cost function for the case considering 1 mode has converged faster than that of 3 modes, and the convergence rate of the case in which 5 vibration modes are considered is the slowest amongst the three plots. The reason lying behind this fact is that the input frequency data are contaminated with measurement noise; hence, the associated effects of this phenomenon are more clearly seen when a larger amount of data are utilized in the damage diagnosis process. Despite the fact that measurement noise can affect the convergence rate of the algorithm in speed, the accuracy of the results remains high.

Eventually, not only are the frequencies polluted with $5 \%$ noise, but the mode shapes are also contaminated with $1 \%$ noise. On this occasion, the first 5 modes of the shear frame are taken into consideration since more noise effects are observed when a higher number of modes are used. Hence, if accurate results can be gained with 5 modes, the results considering lower number of modes will definitely be satisfactory. The problem is optimized using the ICA, with selected parameters as follows: number of decades $=500$, number of countries $=200$, and number of initial imperialists $=20$. In this case, the results with and without noise are shown in Figure 7. 


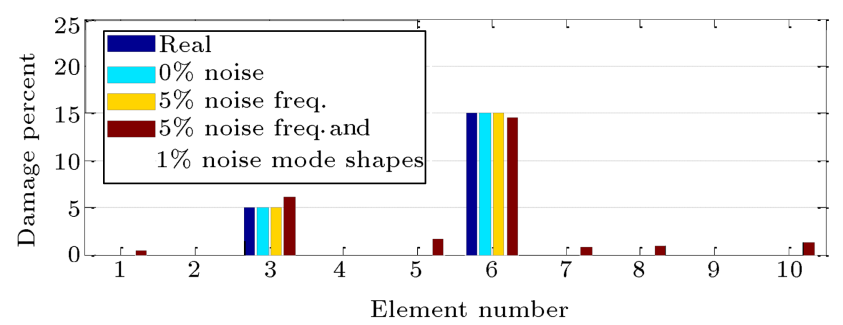

Figure 7. Damage detection results of the shear frame with different levels of noise in $\omega$ and $\phi$.

It is observable from Figure 7 that adding measurement noise to the mode shapes of the structure has led to mistakenly diagnosing some elements with a percentage of damage. However, this false diagnosis is limited to a very low damage level, close to zero, which can be acceptably neglected in damage detection problems. Besides, the level of preciseness of damage diagnosis associated with noisy frequency and mode shape data is accompanied by a negligible amount of discrepancy which is, again, admissible in damage detection problems.

The above figures clearly state that accuracy of the results is not considerably dependent on whether measurement noise exists or not. In fact, considering measurement noises both in natural frequencies and in mode shapes does not weaken the results and the results enjoy a high level of accuracy.

\subsection{Simply supported beam}

In the second example, a ten-element simply supported beam, as shown in Figure 8, is investigated. The beam is $5 \mathrm{~m}$ in length, $0.5 \mathrm{~m}$ in width, and $0.7 \mathrm{~m}$ in height, and is discretized into 10 elements. Modulus of elasticity of the beam is $E=2.5 \times 10^{10} \mathrm{~N} / \mathrm{m}^{2}$, and mass density is $\rho=2500 \mathrm{~kg} / \mathrm{m}^{3}$. The beam is investigated under two different damage scenarios as listed in Table 2. In order to make the results more compatible with real circumstances, the second scenario is investigated under a noisy circumstance. The results are included in what follows. Furthermore, as in the previous example, the results are attained in

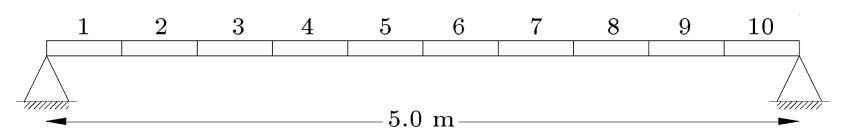

Figure 8. Simply supported beam.

Table 2. Damage scenarios for the simply-supported beam.

\begin{tabular}{ccc}
\hline Scenario & $\begin{array}{c}\text { Element } \\
\text { number }\end{array}$ & $\begin{array}{c}\text { Damage } \\
\text { percent }\end{array}$ \\
\hline 1 & 3 & 10 \\
2 & 4 & 10 \\
2 & 7 & 30 \\
\hline
\end{tabular}

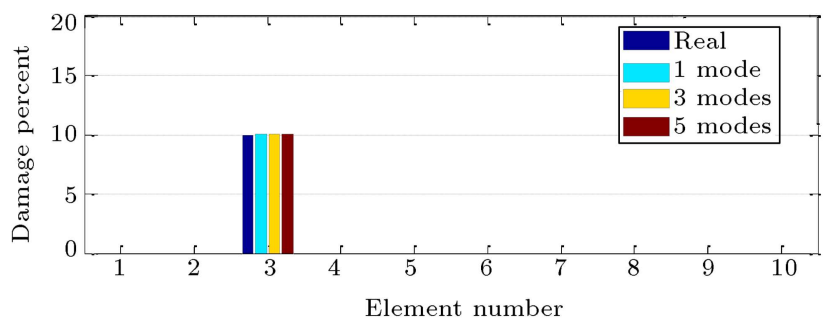

Figure 9. Damage detection results of the first scenario without noise, for simply supported beam.

three cases of the first 1,3 , and 5 vibration modes of the beam so that the efficiency of the proposed method can be lucidly demonstrated.

In the first scenario, $10 \%$ damage is assumed for element number 3 . The problem is then untangled using the ICA, with selected parameters as follows: number of decades $=1000$, number of countries $=200$, and number of initial imperialists $=20$.

Damage detection results using the first 1, 3 and 5 modes of the beam are shown in Figure 9.

The figure vividly illustrates that the exact values of assumed damages for the element are obtained via the propounded method.

The second scenario consists of a multiple damage case: $10 \%$ damage in the fourth element and rather severe 30-percent damage in the 7th element. As mentioned above, to further make the conditions compatible with real cases, natural frequencies of the beam are contaminated with different levels of noise. Therefore, $5 \%, 10 \%, 15 \%$, and $20 \%$ noises are imposed on the frequencies. The problem is then optimized using the ICA, with selected parameters as follows: number of decades $=1000$, number of countries $=$ 200 , and number of initial imperialists $=20$. The convergence curves for this case are shown in Figure 10.

According to the above figure, the convergence rate of the algorithm is vividly affected by the level of noise since whenever the intensity of contamination is escalated, more complexity is added to the search space in the solution domain. Thereupon, the convergence curve corresponding to the case in which $5 \%$ frequency

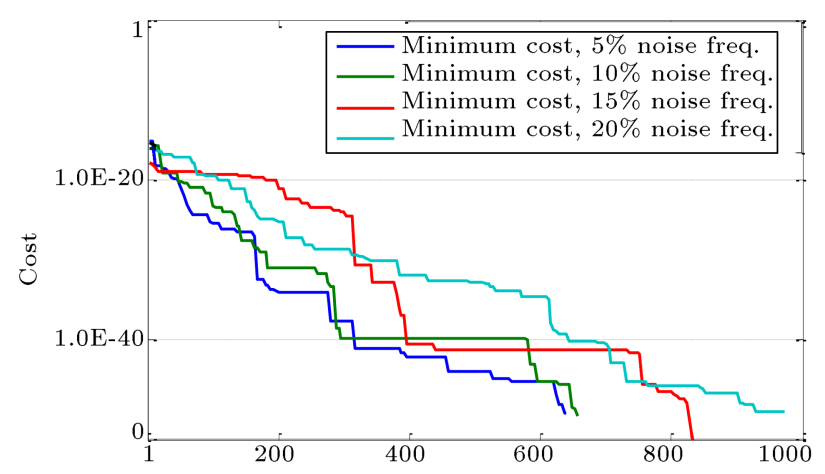

Figure 10. Cost function value versus number of iteration. 


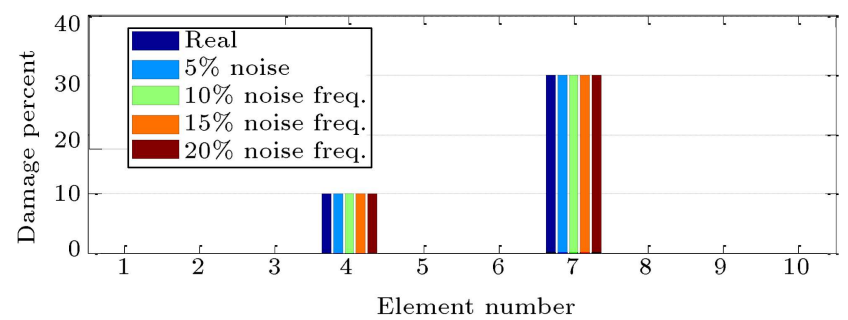

Figure 11. Damage detection results of the second scenario with different levels of noise, for simply supported beam.

noise is imposed possesses the fastest convergence speed since the level of contamination is far lower than that of the other three cases. Damage detection results using the first 3 modes of the beam for this case are shown in Figure 11.

As expected, the results are in good agreement with the real damage severities. Actually, exact values are reaped for damage severities and locations no matter what the number of vibration modes considered is. In fact, even the level of noise applied to the computations has not affected the accuracy of the results; the location and severity of damage in different scenarios is detected with high accuracy since, as it was mentioned earlier, the objective function is highly sensitive to structural damages and relatively insensitive to measurement noise. This characteristic of the offered objective function is highly valuable in solving real-world damage diagnosis problems.

The method, thus, has successfully detected the assumed damage regardless of the number of modes considered, both in noiseless and noisy states.

\subsection{Clamped beam}

Although there are various evolutionary optimization algorithms, all of them follow the same searching rules, and despite the fact that all of them are different in some details, they should all give similar results to a specific problem of interest. This section is assigned to examining a beam via four different optimizers, i.e. ICA, Cuckoo Search (CS), Genetic Algorithm (GA), and pattern search, each of which is an evolutionary search system. It is worth noting again that all evolutionary optimizers have a similar search approach, and only minor differences exist in the details of their search processes as well as their approach to improving the results in different iterations. The structure under consideration is selected to be a 10-element beam with clamped supports at both ends, as shown in Figure 12. As in Section 3.2, the beam is $4 \mathrm{~m}$ in length, $0.5 \mathrm{~m}$ in width, and $0.7 \mathrm{~m}$ in height, and modulus of elasticity

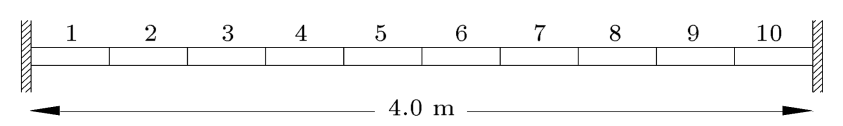

Figure 12. Clamped beam with ten elements.
Table 3. Damage scenarios for the clamped beam.

\begin{tabular}{ccc}
\hline Scenario & $\begin{array}{c}\text { Element } \\
\text { number }\end{array}$ & $\begin{array}{c}\text { Damage } \\
\text { percent }\end{array}$ \\
\hline \multirow{2}{*}{1} & 3 & 15 \\
& 7 & 15 \\
2 & 2 & 10 \\
2 & 5 & 30 \\
& 8 & 20 \\
\hline
\end{tabular}

of the beam is $E=2.5 \times 10^{10} \mathrm{~N} / \mathrm{m}^{2}$, and mass density is $\rho=2500 \mathrm{~kg} / \mathrm{m}^{3}$.

Two damage scenarios as listed in Table 3 are investigated for the beam through the mentioned optimizers.

In addition to the conditions mentioned above, natural frequency measurements are polluted with $5 \%$ noise in both scenarios in order that a more realistic model of the structure can be achieved. The problem is solved using the first five modes of the structure.

The problem is optimized with the four abovementioned optimization algorithms, and damage detection results for the first and second scenarios using these algorithms are given In Figures 13 and 14, respectively.

It is obvious from the bar graphs In Figures 13 and 14 that all four methods have been able to detect the assumed damages. However, whilst ICA has been able to accurately detect the damaged elements without mistakenly detecting any other damaged locations, CS algorithm has been shown to have some minor discrepancies in detecting the damaged elements. To be clearer, a negligible difference can be seen between

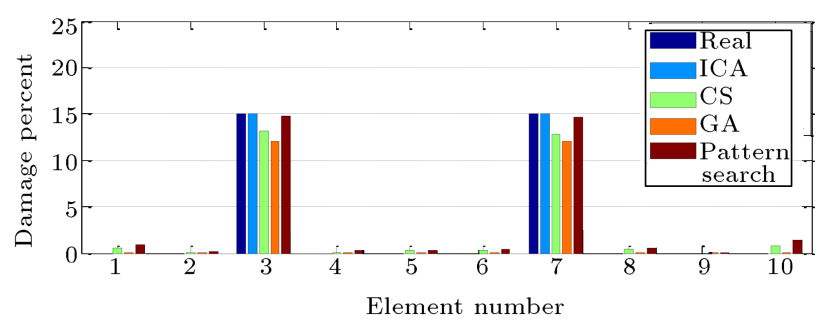

Figure 13. Damage detection results of the first scenario with $5 \%$ noise in $\omega$ using first 5 modes, for clamped beam.

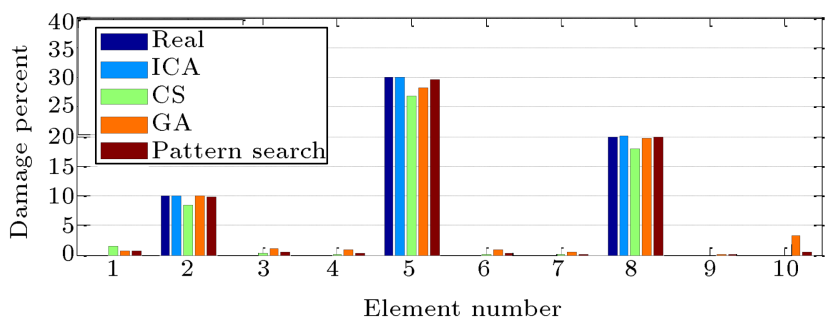

Figure 14. Damage detection results of the second scenario with $5 \%$ noise in $\omega$ using first 5 modes, for clamped beam. 
the real damage quantities and the identified damage severities obtained by CS. In addition, some elements are erroneously identified as damaged even though they are intact. However, the amount of discrepancy in the results can be negligible in comparison with the damage quantities. GA, on the other hand, has successfully detected the damaged locations with lower discrepancy, as compared to CS; however, the damage percentage is mistakenly attributed to some of the intact elements possessing higher values. Besides, pattern search has shown a performance superior to the performances of CS and GA since the results are of higher accuracy. However, the results obtained via ICA are the most reliable of all four algorithms. It should be mentioned that the minor discrepancies observed in CS, GA, and pattern search might be due to either measurement noise effects or their searching parameters.

\subsection{Planar truss}

This section is aimed at examining a steel plane truss as shown in Figure 15 which is taken from [43]. The truss consists of 29 elements as shown in the figure, and the material properties are as follows: modulus of elasticity is $E=200 \mathrm{GPA}$, and mass density is $\rho=$ $7850 \mathrm{~kg} / \mathrm{m}^{3}$. Cross-sectional area and mass per unit length of the horizontal elements at the bottom are $A=0.010 \mathrm{~m}^{2}$ and $m=3000 \mathrm{~kg} / \mathrm{m}$, respectively; those for the horizontal elements at the top are $A=0.010$ $\mathrm{m}^{2}$ and $m=78.5 \mathrm{~kg} / \mathrm{m}$, respectively; those for the vertical elements are $A=0.005 \mathrm{~m}^{2}$ and $m=39.25$ $\mathrm{kg} / \mathrm{m}$, respectively; those for the diagonal elements are $A=0.008 \mathrm{~m}^{2}$ and $m=62.8 \mathrm{~kg} / \mathrm{m}$, respectively [43] .

Two damage scenarios are assumed for this truss, which are listed in Table 4.

As it was done for the previous examples, effects of measurement noises are considered, and $5 \%$ noise is imposed on the natural frequencies in the first scenario. Damage is detected in three cases of the first 1,3 , and 5 modes of the truss in this scenario in order that effects

Table 4. Damage scenarios for the plane truss.

\begin{tabular}{ccc}
\hline Scenario & $\begin{array}{c}\text { Element } \\
\text { number }\end{array}$ & $\begin{array}{c}\text { Damage } \\
\text { percent }\end{array}$ \\
\hline 1 & 5 & 10 \\
& 2 & 30 \\
2 & 19 & 20 \\
& 28 & 10 \\
\hline
\end{tabular}

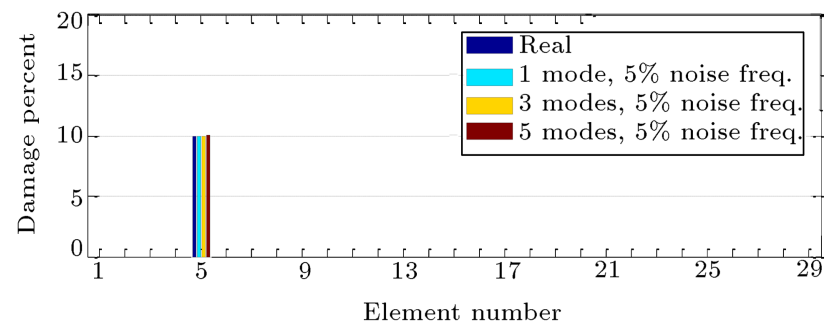

Figure 16. Damage detection results of the scenario with $5 \%$ noise in $\omega, 29$-element planar truss.

of the number of vibrational modes under consideration can be observed in the results. The problem is optimized using the ICA, with selected parameters as follows: number of decades $=1000$, number of countries $=200$, number of initial imperialists $=20$, and the results attributed to this part are shown in Figure 16.

As expected, damage is successfully detected in the presence of measurement noises, considering different vibration modes in the calculations.

As it was noted in the previous section, although all evolutionary algorithms have similar mechanisms in finding the optimum solutions and, thus, their answers to the same problem should not be widely different, there always are some differences due to their divergences in details. Moreover, in damage detection problems, it is important to use an optimizer the solutions of which to a certain problem in different runs are in a specific admissible range. In other words, the stability of the solutions is what makes an optimization algorithm different from others since, as shown in the previous examples, the proposed method of this paper is capable of detecting damage, independent of the optimization algorithm selected to solve the inverse problem. Thus, in this section, a comparison is made between four evolutionary algorithms of ICA, GA, pattern search, and CS so as to compare their stability statuses in repeated runs. This is done in order to single out the optimization algorithm which results in the most stable solution trend in different runs. Accordingly, a plot of five consecutive runs for each algorithm is provided in this part for solving the damage detection problem of the second scenario of the truss.

As shown in Table 4, it is assumed that element no. 2 received $30 \%$ damage, the 19 th element received $20 \%$ damage, and the 28th element contained $10 \%$ damage. In addition, both frequencies and mode

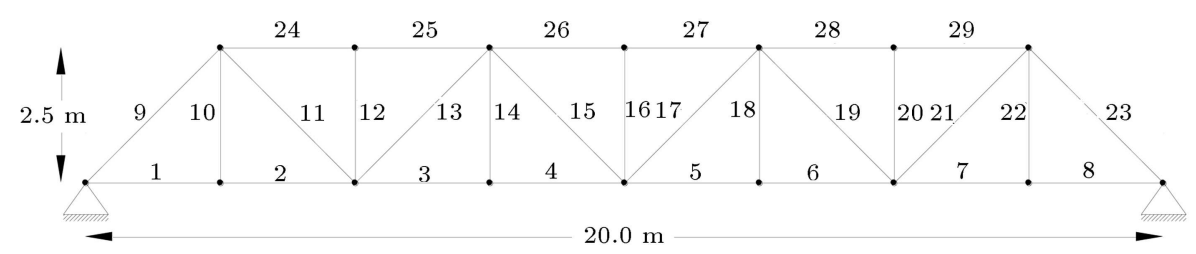

Figure 15. 29-element planar truss [43]. 


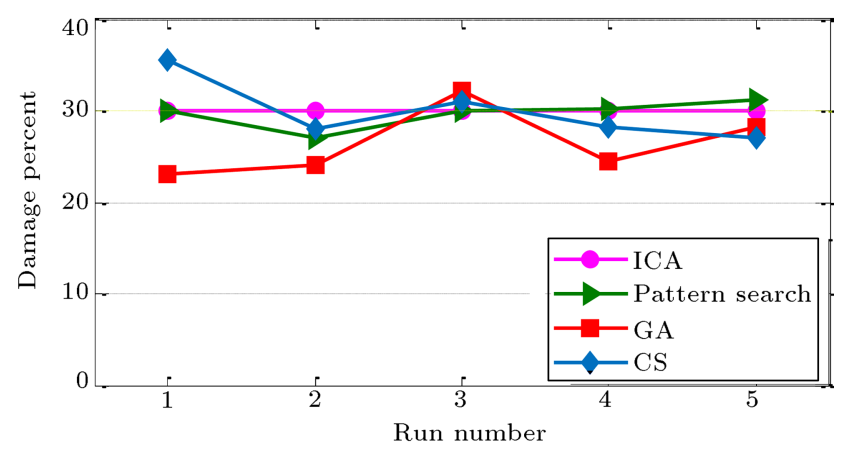

Figure 17. Damage detection results in 5 runs for element no. 2 using ICA, GA, pattern search, and CS, with $5 \%$ noise in $\omega$ and $5 \%$ noise in $\phi$.

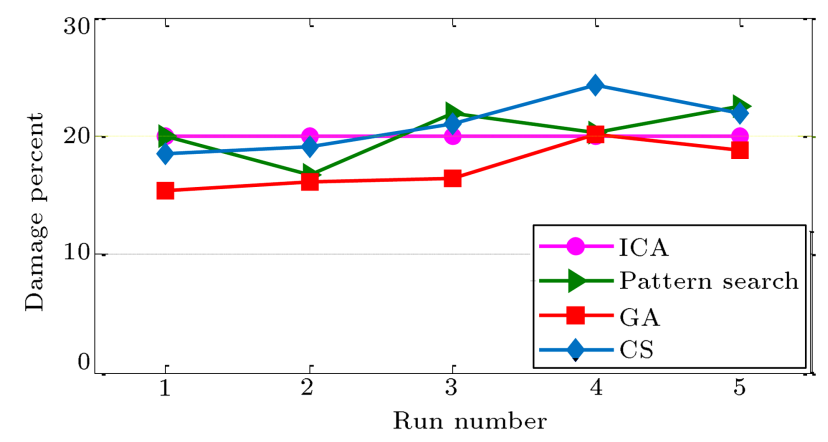

Figure 18. Damage detection results in 5 runs for element no. 19 using ICA, GA, pattern search, and CS, with $5 \%$ noise in $\omega$ and $5 \%$ noise in $\phi$.

shapes are polluted with $5 \%$ noise so as to make the situation more similar to the real conditions, and the problem is solved using the first five modes of the structure. Then, each of the above-mentioned optimizers is used five times to solve the inverse problem of damage detection for this case. Figure 17 shows the results of these algorithms in the five runs performed for detected damage severity of element no. 2, and the yellow line illustrates the real damage value.

Damage detection results using the four optimization algorithms in five runs for the 19th element are shown in Figure 18.

The results obtained from the same procedure for the 28th element are shown in Figure 19.

Although all algorithms have been able to estimate the damage severity in the selected damaged element, it is clear from the figure that not every algorithm can reach a unique value in all runs. ICA seems to have the most stable trend of solutions among all of the utilized algorithms since its solutions in five runs are approximately the same. This demonstrates that ICA is a stable algorithm which can be trusted in searching the optimum solution to the damage detection scheme proposed in this paper.

That all of the algorithms have been able to approximately estimate damage is an illustration of the robustness of the intended damage detection method;

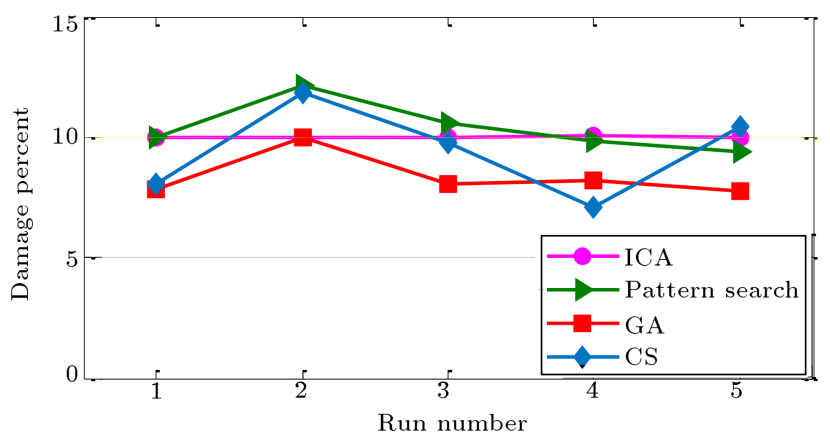

Figure 19. Damage detection results in 5 runs for element no. 28 using ICA, GA, pattern search, and CS, with $5 \%$ noise in $\omega$ and $5 \%$ noise in $\phi$.

further, accurate results are obtained with the selection of ICA as the optimization tool to solve the inverse problem of damage detection.

\section{Conclusion}

An effective damage identification method was developed in this paper in pursuit of diagnosing and localizing existing damages. Additionally, this paper put forward a novel method utilizing the modal assurance criterion in which natural frequencies and modal shapes were obtained from the characteristic equation of the structure in free vibration, and then were used separately as the parameters required in MAC formula. An effective objective function was also suggested based on MAC with the imperialist competitive algorithm as the optimization method to solve the damage detection problem.

The method was validated using four different numerical examples, and a comparison between the ICA algorithm and three other algorithms was made to better show the efficiency of the technique and the selected optimization scheme. The results demonstrate clearly the effectiveness of the method in detecting both the severity and location of the existing damages. Another striking point which is lucidly observed throughout the paper is the high stability of the selected optimizer, i.e. ICA, while obtaining the minimum solution to the damage detection problem which is recognized via the comparative numerical investigations of the paper to be the best fitted optimization algorithm for the proposed method. This characteristic makes the whole proposed damage detection procedure reliable enough to be implemented on real-life structures.

\section{References}

1. Farrar, C.R. and Worden, K. "An introduction to structural health monitoring", Phil. Trans. R. Soc. A., 365(1851), pp. 303-315 (2007).

2. Yan, Y.J., Cheng, L., Wu, Z.Y., and Yam, L.H. "Development in vibration-based structural damage 
detection techniques", Mech sys signal process, 21(5), pp. 2198-2211(2007).

3. Shi, Z.Y., Law, S.S., and Zhang, L.M. "Structural damage detection from modal strain energy change", J. Eng Mech ASCE, 126(12), pp. 1216-1223 (2000).

4. Gawronski, W. Sawicki, J.T. "Structural damage detection using modal norms", J. Sound Vib, 229(1), pp. 194-198 (2000).

5. Kawiecki, G. "Modal damping measurement for damage detection", Smart Mat. Struct, 10(3), pp. 466-471 (2001).

6. Shi, Z.Y., Law, S.S., and Zhang, L.M., "Improved damage quantification from elemental modal strain energy change", J. Eng. Mech-ASCE, 128(5), pp. 521529 (2002).

7. Abdo, M.A.B. and Hori, M. "A numerical study of structural damage detection using changes in the rotation of mode shapes", J. Sound. Vib., 251(2), pp. 227-239 (2002).

8. Sampaio, R.P.C., Maia, N.M.M., and Silva, J.M.M. "The frequency domain assurance criterion as a tool for damage detection, damage assessment of structures", Proceedings Key Eng. Mat., 245, pp. 69-76 (2003).

9. Doebling, S.W., Farrar, C.R., and Prime, M.B. "A summary review of vibration-based damage identification methods", Shock Vib. Dig., 30(2), pp. 91-105 (1998).

10. Yan, Y.J., Cheng, L., Wu, Z.Y., and Yam, L.H. "Development in vibration-based structural damage detection technique", Mech. Sys. Signal Process., 21(5), pp. 2198-2211 (2007).

11. Fang, S.E., Perera, R, De., and Roeck, G. "Damage identification of a reinforced concrete frame by finite element model updating using damage parameterization", J. Sound. Vib., 313(3). pp. 544-559 (2008).

12. Tabrizian, Z., Ghodrati Amiri, G., and Hossein Ali Beygi, M. "Charged system search algorithm utilized for structural damage detection", Shock Vib., 13 pages (2014).

13. Gau, H.Y. and Li, Z.L. "Structural damage identification based on Bayesian theory and improved immune genetic algorithm", Expert Syst. Appl., 39(7), pp. 6426-6436 (2012).

14. Salawu, O.S. "Detection of structural damage through changes in frequency: a review", Eng Struct, 19(9), pp. 718-723 (1997).

15. Ren, W.X. and De Roeck, G. "Structural damage identification using modal data. I: Simulation verification", J. Struct. Eng. ASCE, 128(1), pp. 87-95 (2002).

16. Ren, W.X. and De Roeck, G. "Structural damage identification using modal data. II: Test verification", J. Struct. Eng. ASCE, 128(1), pp. 96-104 (2002).

17. Abdel Wahab, M.M. and De Roeck, G. "Damage detection in bridges using modal curvatures: application to a real damage scenario", J. Sound. Vib., 226(2), pp. 217-235 (1999).
18. Shi, Z.Y., Law, S.S., and Zhang, L.M. "Structural damage detection from modal strain energy change", J. Eng. Mech. ASCE, 126(12), pp. 1216-1223 (2000).

19. Jaishi, B. and Ren, W.X. "Structural finite element model updating using ambient vibration test results", J. Struct. Eng. ASCE, 131(4), pp. 617-628 (2005).

20. Jaishi, B. and Ren, W.X. "Damage detection by finite element model updating using modal flexibility residual", J. Sound. Vib., 290(1). pp. 369-387 (2006).

21. Jaishi, B. and Ren, W.X. "Finite element model updating based on eigenvalue and strain energy residuals using multiobjective optimisation technique", Mech. Sys. Signal Process, 21(5), pp. 2295-2317 (2007).

22. Jaishi, B., Kim, H.J., Kim, M.K., Ren, W.X., and Lee, S.H. "Finite element model updating of concrete-filled steel tubular arch bridge under operational condition using modal flexibility", Mech. Sys. Signal Process, 21(6), pp. 2406-2426 (2007).

23. Perera, R. and Ruiz, A. "A multistage FE updating procedure for damage identification in large-scale structures based on multiobjective evolutionary optimization", Mech. Sys. Signal Process, 22(4). pp. 970991 (2008).

24. Kaveh, A. and Talatahari, S. "Optimum design of skeletal structures using imperialist competitive algorithm", Comput. struct., 88(21), pp. 1220-1229 (2010).

25. Kaveh, A. and Zolghadr, A. "An improved CSS for damage detection of truss structures using changes in natural frequencies", Advances in Eng. Soft., 80, pp. 93-100 (2015).

26. Saada, M., Arafa, M., and Nassef, A. "Finite element model updating approach to damage identification in beams using particle swarm optimization", Eng. Opt., 45(6), pp. 677- 696 (2013).

27. Kaveh, A., Javadi, S.M. and Maniat, M. "Damage detection via modal data with a mixed particle swarm strategy, ray optimizer, and harmony search", Asian J. Civil Eng. (BHRC), 15(1), pp. 95-106 (2014).

28. Zare Hosseinzadeh, A., Bagheri, A., and Ghodrati Amiri, G. "Two-stage method for damage localization and quantification in high- rise shear frames based on the first mode shape slope", Int. J. Optim. Civil Eng., 3(4), pp. 653-672 (2013).

29. Pandey, A.K., Biswas, M., and Samman, M.M., "Damage detection from changes in curvature mode shapes", J. Sound Vib., 145(2), pp. 321-332 (1991).

30. Moreno-Garcıa, P., Dos Santos, J.V.A., and Lopes. H., "A new technique to optimize the use of mode shape derivatives to localize damage in laminated composite plates", Composite Struct, 108, pp. 548-554 (2014).

31. Pandey, A.K. and Biswas, M., "Damage detection in structures using changes in flexibility", J. Sound Vib., 169(1), pp. 3-17 (1994).

32. Balsamo, L., Mukhopadhyay, S., and Betti. R., "A statistical framework with stiffness proportional damage sensitive features for structural health monitoring", Smart Struct. Sys., 15(3), pp. 699-715 (2015). 
33. Dos Santos, J.V.A., Soares, C.M.M., Soares, C.A.M., and Pina, H.L.G., "A damage identification numerical model based on the sensitivity of orthogonality conditions and least squares techniques", J. Comput. Struct., 78(1), pp. 283-291 (2000).

34. Perera, R., Fang, S. and Huerta, C. "Structural crack detection without updated baseline model by single and multiobjective optimization", Mech. Sys. Signal Process., 23(3), pp. 752-768 (2009).

35. Nouri Shirazi, M.R. and Mollamahmoudi, H. "Structural damage identification using an adaptive multistage optimization method based on a modified particle swarm algorithm", J. Optim. Theory Appl., 160(3), pp. 1009-1019.

36. Yu, L. and Fu, Y. "Structural damage detection by fusion of GA and PSO", Advanced Mat. Research, 919, pp. 338-343 (2014).

37. Kaveh, A. and Maniat, M. "Damage detection based on MCSS and PSO using modal data", Smart Struct. Sys., 15(5), pp. 1253-1270 (2015).

38. Seyedpoor, S.M. and Yazdanpanah, O. "Structural damage detection by differential evolution as a global optimization algorithm", Iranian J. Struct. Eng., 1(1), pp. 52-62 (2015).

39. Xu, H.J., Liu, J.K., and Lv, Z.R. "Structural damage identification based on modified cuckoo search algorithm", Struct. Eng. Mech., 58(1), pp. 163-179 (2016).

40. Allemang, R.J. and Brown D.L., "A correlation coefficient for modal vector analysis", Proceed. 1st Int. Modal. Analysis Conf., Kissimmee, FL, USA, 1, pp. 110-116 (1982).

41. Atashpaz-Gargari, E. and Lucas, C. "Imperialist competitive algorithm: an algorithm for optimization inspired by imperialistic competition", IEEE Cong Evolutionary Comput, Singapore, pp. 4661-4667 (2007).

42. Ghodrati Amiri, G., Zare Hosseinzadeh, A., Bagheri, A., et al. "Damage prognosis by means of modal residual force and static deflections obtained by modal flex- ibility based on the diagonalization method", Smart Mter. Struct., 22(7), 075032 (2013).

43. Ghodrati Amiri, G., Zare Hosseinzadeh, A., and Seyed Razzaghi, S.A. "Generalized flexibility- based model updating approach via democratic particle swarm optimization algorithm for structural damage prognoisis", Int. J. Optim. Civil Eng., 5(4), pp. 445-464 (2015).

\section{Biographies}

Hooshang Dabbagh is currently an Assistant Professor in the University of Kurdistan. He received his $\mathrm{BSc}, \mathrm{MSc}$ and $\mathrm{PhD}$ degrees in Civil Engineering from Sharif University of Technology, Structural Engineering from Iran University of Science and Technology, and Structural Engineering from University of New South Wales (Australia), respectively. His research interests include the numerical analysis of concrete structures, the rehabilitation of concrete structures using FRP composites and structural health monitoring.

Gholamreza Ghodrati Amiri received his BSc and MSc degrees in Civil Engineering from Sharif University of Technology, Iran, and his $\mathrm{PhD}$ degree in Structural and Earthquake Engineering from McGill University, Canada. He is currently a Professor at Iran University of Science and Technology. His research interests include seismic hazard analysis, performance based earthquake engineering, and structural health monitoring.

Shilan Shaabani received her BSc degree in Civil Engineering from University of Kurdistan, Iran in 2011. She pursued MSc degree in 2011 and received it in Structural Engineering from University of Kurdistan in 2014. Her research interests are structural health monitoring and non-destructive testing of structures with emphasis on damage detection. 